\title{
Análisis del perfil sociodemográfico de la mujer como participante en raids de aventura
}

\section{Social-demographic analysis of raids adventure sportswomen}

\author{
*Antonio Baena Extremera, ${ }^{* *}$ Socorro Rebollo Rico \\ *Universidad de Murcia y **Universidad de Granada (España)
}

\begin{abstract}
Resumen: El objetivo de este trabajo es determinar el perfil sociodemográfico de las mujeres que practican Raids de Aventura en España. La muestra elegida corresponde a la población que participa en la Liga Española de Raids de Aventura, seleccionando las cuatro pruebas más importantes. Hemos utilizando como técnica de obtención de datos la encuesta y como instrumento el cuestionario, aplicado antes de cada competición, durante el briefing. El diseño del trabajo es no experimental, descriptivo y seccional. En el estudio de validez del cuestionario hemos aplicado el coeficiente Rho de Spearman y el coeficiente Alfa de Crombach. Y para la obtención de los resultados, hemos aplicado a las variables, estadística descriptiva. Podemos afirmar que el perfil coincide con una mujer de 26-35 años, poco practicante, soltera, con estudios universitarios, autónomas, y con trabajos de asalariadas o docentes.
\end{abstract}

Palabras clave: perfil sociodemográfico, mujer, deportes de aventura, raids de aventura.

\begin{abstract}
The goal of this study is to determine the social-demographic profile of women who practise adventure competitions. The chosen sample corresponds to the population participating in Spanish Ligue of Adventure Raids 2004, by selecting the four most important challenges. The technique used to obtain the data sample is the survey and the applied questionnaire our working tool, while briefing before each competition. The work design is no experimental, descriptive and secional. For the estability of questionnaire, we have used the Spearman rho-parameter and Crombach alphaparameter. To obtain the results we have applied to our variables descriptive statistics. We can assure that the profile corresponds with a woman 26 to 35 aged not very practising, single, with higher degree studies, independent with own economic resources on teachers.
\end{abstract}

Keywords: Social-demographic profile, women, adventure sports, adventure, raids.

\section{Introducción}

Queremos introducir este trabajo con una cita de Pinos (1997), que afirma que la naturaleza, el mayor campo de deportes del mundo, nos regalará siempre nuevos espacios y situaciones para la práctica deportiva. Citamos esta reflexión porque consideramos que define de forma clara elámbito de actuación de esta investigación: el medio natural $\mathrm{y}$ las diferentes tendencias deportivas que en este se ubican.

Podemos entender que el espacio natural del s.XXI, es un espacio deportivo, donde las actividades relacionadas con la aventura y el riesgo son las principales protagonistas. La tecnología ha facilitado los recursos e instrumentos pertinentes para el desarrollo de estas actividades recreativas, que nos permiten deslizarnos por el agua, el aire y la tierra, provocando notables sensaciones de placer, riesgo y aventura en función de las expectativas de cada cual (Rebollo y Feriche, 2002).

La capacidad de este entorno y de este tipo de prácticas para causar sensaciones y vivencias fuera de la cotidianidad, es uno de los mayores motivos del desarrollo de los deportes de aventura (DA). En esta línea, Martos (1999), afirma en su estudio sobre las razones de la práctica de DA por la población joven, que con estas actividades se persiguen las emociones corporales, el riesgo, el desafío y lo improvisto.

Esta aventura y riesgo, antiguamente sólo apta para aquellos «hombres» más arriesgados y valientes, está siendo también objeto de interés de mujeres deportistas y en general de todo tipo de practicantes, sin distinción de género, edad, clase social, etc. Este fenómeno se ve favorecido por la proliferación de empresas que se encargan de acercar estos deportes a toda la población (Sicilia, 1999; Herrera y Pilatti, 2005).

Debido al aumento de participación femenina en las prácticas deportivas de aventura, en los últimos años se ha investigado sobre intereses, motivaciones y tendencias de la práctica deportiva femenina en la naturaleza. Canales, Lagardera y Percih (1995) y Canales y Perich (2000), indagan en las motivaciones y significados de este tipo de prácticas para la mujer. Los autores concluyen que las practicantes utilizan las actividades físicas en la naturaleza como medio de catarsis para

Fecha recepción: 21-02-08 - Fecha envío revisores: 21-02-08 - Fecha de aceptación: 10-06-08 Correspondencia: Antonio Baena Extremera

$\mathrm{C} /$ Amistad $12^{\circ} \mathrm{B}$

04800 Albox (Almería)

E-mail: abaenaextrem@yahoo.es evadirse de la vida cotidiana, para encontrarse a sí mismo, para sentirse bien y superarse cada vez más a uno mismo.

En una encuesta sobre la practica deportiva en la naturaleza desde una perspectiva turística en la ciudad de Barcelona, se detalló que el $52 \%$ de las mujeres encuestadas manifestaron que estar en contacto con la naturaleza representaba su mayor interés, seguido por un $26 \%$ que manifestaban hacerlo por diversión y un $10 \%$ por evadirse de la rutina cotidiana (Olivera y Olivera, 1998, p.79).

Pese a esta pluralidad de los DA, la inclusión de la mujer en este tipo de actividades ha sido más tardía, destacando la menor participación de la mujer en los DA frente a la participación del hombre. Esto es un reflejo de lo que sigue ocurriendo en la práctica deportiva general por parte de la población. No obstante, Baigorria y Fernández (1996), pasaron 502 encuestas a una población de cuatro municipios de Badajoz, y destacaron que el senderismo es uno de los deportes más demandados especialmente por la población femenina.

Cabe destacar los Raids de Aventura (RA) como la forma más pura de practicar los DA, puesto que se trata de una competición que aúna muchas modalidades deportivas, impregnándolas de aventura, riesgo, esfuerzo y autosuperación. Las competiciones de aventura son pruebas multidisciplinares de larga duración o ultrafondo que se celebran en entornos naturales y que incluyen la práctica sin apenas descanso y bajo el marco de la orientación de actividades outdoor como: Carrera a pie, trekking, bicicleta de montaña, orientación, escalada, descenso de barrancos, kayak, tiro con arco, esquí de montaña, rafting, etc.

Desde la perspectiva del género, la participación de la mujer en estas competiciones plantean muchos interrogantes: de tipo organizativo, rendimiento, sociodemográfico, etc. Por este motivo, el interés por conocer los perfiles deportivos y sociodemográfico de las participantes es cada vez mayor.

Roca (2005), entiende que los raids son de las pocas competiciones, por no decir la única, en que en un mismo equipo hay hombres y mujeres mezclados y en que la clasificación general está formada por equipos mixtos. En el reglamento de los raids internacionales, un equipo tiene que tener como mínimo un componente del sexo opuesto.

Miguel, Andrade y Portela (2005), en un estudio sobre las características del perfil socio-económico de los competidores de carreras de aventura, llegan a la conclusión de que el perfil de estos competidores es de 19 a 43 años de edad, siendo la mayoría hombres, solteros, con un 


\begin{tabular}{|c|c|c|}
\hline VARIABLE & DIMENSIONES & INDICADORES \\
\hline \multirow{9}{*}{$\begin{array}{l}\text { Perfil sociodemográficos de } \\
\text { la mujer como participante en } \\
\text { raids de aventura. }\end{array}$} & Datos de Identificación, & Edad, género, estado civil \\
\hline & Nivel y tipo de Instrucción & $\begin{array}{l}\text { Sin estudios, estudios primarios, estudios secundarios, } \\
\text { formación profesional, diplomados universitarios, } \\
\text { universitario superior, otros, relación con deporte y medio } \\
\text { ambiente. }\end{array}$ \\
\hline & Actividad Económica & $\ldots$ \\
\hline & Situación actual & $\begin{array}{l}\text { Estudiante, estudia y trabaja, busca su primer empleo, } \\
\text { activo ocupado, activo parado, labores del hogar, } \\
\text { jubilado/pensionista, servicio militar, otros. }\end{array}$ \\
\hline & Participación masculina y femenina & $\mathrm{Si}$, no \\
\hline & Motivos & $\begin{array}{l}\text { Por la organización, por la dificultad técnicas, por la } \\
\text { dificultad táctica, por la dificultad física, por la dificultad } \\
\text { psicológica, por motivos de cultura deportiva, por la } \\
\text { educación deportiva de la mujer, otras. }\end{array}$ \\
\hline & Importancia de la participación femenina & $\begin{array}{l}\text { Muy importante, bastante importante, importante, poco } \\
\text { importante, nada importante. }\end{array}$ \\
\hline & Aspectos & $\begin{array}{l}\text { Nivel físico, nivel táctico, nivel técnico, nivel psicológico, } \\
\text { otros. }\end{array}$ \\
\hline & Requisitos de la mujer raider. & Igual que el raider masculino, diferentes. \\
\hline
\end{tabular}

trabajo de 5 a 8 horas de media, y con unos niveles de estudios de segundo grado.

En relación a los aspectos competitivos, Martos (2004), tiene la convicción de que las mujeres que compiten en RA, tienen un mayor rendimiento en las pruebas de orientación y de aguante y menos en las pruebas que exigen explosividad, y que cuando se encadenan un par de secciones de fuerza, suelen ir quedándose atrás los equipos femeninos y mixtos.

Roca (2005), expresa que un equipo únicamente masculino haría las carreras mucho más rápidas, más duras, más interminables y con muchos más abandonos. Arias (2005), opina que siempre se ha dicho que la mujer en un raid juega un papel decisivo, porque en ella reside la fuerza mental y la estrategia de equipo

En este sentido Bustillo (2005) opina, que hay que animar a las mujeres. Las chicas son menos explosivas pero tienen mucha fuerza psicológica, dosifican a la perfección su esfuerzo y frente a los chicos, superan situaciones y distancias en mejores condiciones.

Comentar que dentro de estas pruebas tan duras existen raids únicamente para mujeres, como el «Women Internacional Adventure Raid Salomón» (W.I.A.R.) o el «Raid Wiar Salomón de Catalunya» y otros raids novedosos actualmente llamados Triraids (Roca, 2005). Sobre el W.I.A.R.,Aguilera (2004), corrobora la dureza de estas pruebas, cuando en sus entrevistas relata las pruebas con secciones de bicicleta de montaña, orientación, trekking, patines en línea, kayak, natación, rappel, esquí de fondo y tirolina.

Dada la importancia que están adquiriendo estas prácticas por la población femenina y la falta de investigaciones sobre los diferentes factores que la definen, consideramos de gran interés conocery analizar: el perfil sociodemográfico de estas practicantes. Y así, de los datos resultantes que hemos obtenido, consideramos que podemos aportar interesantes conclusiones para el desarrollo de esta perspectiva científica y para la mejora de la oferta empresarial, la calidad de la organización de pruebas y competiciones deportivas, como raids.

Nos planteamos como objetivo en este trabajo el analizar y conocer el perfil sociodemográfico de las mujeres que practican RA, para ello, analizaremos las féminas que participan en las competiciones más importantes en España.

\section{Método}

\subsection{Diseño}

Para el presente trabajo, se ha procedido a realizar un diseño no experimental descriptivo y seccional.

\subsection{Universo y Población}

Nuestro universo de estudio es la Liga Española de RA, la cual consta de varias pruebas. Hemos definido como población de estudio, cuatro de ellas: Raid Gredos, Raid Bimbache Extrem, Raid TransAventur, Raid Sierra Espuña, tomando como criterios de elección, la importancia a nivel estatal de la prueba, la localización geográfica, el número de participantes y la fecha de realización. Dentro de estas competiciones, hemos seleccionado como muestra dentro de una población finita y para un error muestral de $\pm 2 \%$, a todos los participantes que compiten dentro de la Liga Española y que no repiten en ninguna de estas cuatro pruebas, siendo el total 272 sujetos de investigación seleccionados como muestra de este trabajo. De los 272 participantes, la muestra de mujeres se compone de 43 personas.

\subsection{Procedimiento de validación y aplicación del cuestiona-} rio

Para la validación del cuestionario, se ha utilizado el Programa estadístico SPSS 13 llevando a cabo por un lado un análisis de estabilidad y por otro lado un análisis de la fiabilidad del mismo para su validación estadística. Para la validación estadística hemos utilizado el coeficiente de correlación de Spearman, que nos informa de la estabilidad del cuestionario. Concretamente hemos realizado las correlaciones de cada item del test con su correspondiente en el retest. Un $90 \%$ de las correlaciones resultaron positivas o significativas, lo que es indicativo de la estabilidad del mismo. De igual forma, se ha hallado el coeficiente alfa de Crombach en el test, obteniéndose valores por encima de 0.70 lo cual indica un alto grado de fiabilidad.

Posteriormente, se ha procedido a la codificación de las respuestas para cada pregunta del cuestionario quedando fijados los items. A continuación, se realizó la tabulación mediante el sistema informático SPSS 13.

Una vez elaborado el cuestionario, se seleccionaron los RA más representantes en nuestro país, fijándonos por tanto en la Liga Española de RA, donde tras concretar la cita con la organización, se acudió para pasar el cuestionario a los participantes. En el art.1.2 del Reglamento de la Liga Española se expresa: puntuarán para la Liga Española de $R A$ 2004 (LERA), cuatro convocatorias de fin de semana en constante lucha contra el cronometro, para equipos de tres raiders y su asistencia, guiadas cartográficamente y por hojas de ruta.

La organización de cada prueba cita a los raiders el día previo a la carrera, donde se debía acudir en horario de 17:30 a 23:30 para entregar 
el recibo del pago de la competición, la hoja de aptitudes físicas y rellenar los últimos formularios. En ese mismo horario y conforme iban llegando los equipos, se les proporcionaba el cuestionario de forma autoadministrada, que iba siendo rellenado grupo por grupo.

\subsection{Variables}

Las variables del estudio de este trabajo son el: perfil sociodemográfico de la mujer como practicante de RA. Estableciendo a ésta sus dimensiones (Tabla 1).

\subsection{Material o Instrumental}

Como técnica de obtención de datos, hemos utilizado la encuestay como instrumento el cuestionario, el cual consta de 11 preguntas.

El tipo de pregunta del cuestionario va a depender del contenido de la cuestión y del tipo de respuesta, por lo que el tipo de preguntas del cuestionario viene determinado por las variables analizadas en el estudio y por las categorías de estas, de forma que las preguntas del instrumento quedan categorizadas en diferentes posibilidades de respuestas. Concretamente en función de estas variables se formulan las preguntas correspondientes a cada una de ellas:

- Preguntas de identificación: edad, estado civil, estudios,...

\begin{tabular}{|c|c|c|c|}
\hline & & $\mathrm{N}$ & $\%$ \\
\hline \multirow{6}{*}{ Edad } & 20 a 25 años & 9 & 20,9 \\
\hline & 26 a 30 años & 15 & 34,9 \\
\hline & 31 a 35 años & 16 & 37,2 \\
\hline & 36 a 40 años & 3 & 7,0 \\
\hline & 41 a 45 años & 0 & 0,0 \\
\hline & 46 a 50 años & 0 & 0,0 \\
\hline \multirow{4}{*}{ Estado civil } & Soltera & 31 & 72,1 \\
\hline & Casada & 4 & 9,3 \\
\hline & Divorciada & 0 & 0,0 \\
\hline & Otras & 8 & 18,6 \\
\hline \multirow{7}{*}{ Nivel de estudios } & Sin estudios & 0 & 0,0 \\
\hline & Primaria & 2 & 4,7 \\
\hline & Secundaria & 3 & 7,0 \\
\hline & Formación profesional & 5 & 11,6 \\
\hline & Diplomado & 6 & 14,0 \\
\hline & Universitario superior & 26 & 60,5 \\
\hline & Otros & 1 & 2,3 \\
\hline \multirow{8}{*}{ Profesión } & Autónomo & 11 & 26,8 \\
\hline & Funcionario & 3 & 7,9 \\
\hline & Docente Actividad Física y EF & 15 & 35,8 \\
\hline & Bombero & 2 & 4,8 \\
\hline & Empresario & 0 & 0,0 \\
\hline & Trabajador asalariado & 10 & 24,7 \\
\hline & Estudiante & 0 & 0,0 \\
\hline & Otros & 0 & 0,0 \\
\hline \multirow{6}{*}{ Situación actual } & Estudiante & 0 & 0,0 \\
\hline & Estudia y trabaja & 15 & 34,9 \\
\hline & Busca su primer empleo & 0 & 0,0 \\
\hline & Activa ocupada & 28 & 65,1 \\
\hline & Activa parada & 0 & 0,0 \\
\hline & Otras & 0 & 0,0 \\
\hline
\end{tabular}

- Preguntas de información: participación femenina, motivos de participación, importancia de la mujer,.. .

Con respecto al tipo de respuesta, en su mayoría son de tipo cerrada y categorizadas y únicamente 1 de tipo abierta que se ha tratado con remodificación automática (Sierra, 2001,p. 308).

\subsection{Procedimiento}

Siguiendo a Gutiérrez y Oña (2005, p. 185), el procedimiento ha sido el siguiente:

1.Definición del objeto de estudio: Establecimiento de fines y objetivos del estudio.

2.Diseño del cuestionario: Por un lado, hemos elaborado las preguntas, seleccionado y organizado el tipo de cuestionario y se ha procedido a seleccionar la muestra. Posteriormente, para la validación estadística hemos utilizado el coeficiente de correlación Rho de Spearman, que nos indica de la estabilidad del cuestionario, obteniendo un $90 \%$ de correlaciones positivas, lo que es indicativo de la estabilidad del mismo. Para comprobar la fiabilidad, se ha aplicado el coeficiente Alfa de Crombach, obteniéndose valores por encima de 0.70 , lo cual indica el alto grado de fiabilidad.

3.Aplicación del cuestionario a la muestra definida durante el Briefing de cada prueba.

4.Tratamiento de los datos: Revisión de la aplicación del cuestionario, codificación y tabulación.

5.Análisis de los datos: Preparación de la base de datos y análisis estadístico de los datos a través del programa SPSS versión 13 para Windows.

6.Informe final: Interpretación de los datos estadísticos resultantes y elaboración del informe final.

\section{Resultados}

\subsection{Edades de los practicantes}

Las edades de las mujeres practicantes, se sitúan con una frecuencia de $20,9 \%$ en edades de 20 a 25 años, mientras que para el rango de 26 a 31 se sitúa en un $34,9 \%$. Entre los 31 a 35 años, representan el 37,2\%, y entre los 36 y 40 años sólo hay un 7,0\%. Las mujeres, a partir de los 41 años, ya no practican RA.

\subsection{Género de los competidores}

Con respectos al género de los competidores, taly como se puede ver en la tabla 3 , las mujeres representan el 15,5\%, siendo un dato bastante inferior al de los hombres con $84,5 \%$.

\begin{tabular}{|c|c|c|}
\hline \multicolumn{3}{|c|}{ Tabla 3: Género de los raiders } \\
\hline & $\mathbf{N}$ & $\mathbf{\%}$ \\
\hline Mujer & 43 & 15,5 \\
\hline Hombre & 229 & 84,5 \\
\hline Total & 272 & 100,0 \\
\hline
\end{tabular}

\subsection{Estado civil de las practicantes}

El estado civil de las mujeres es principalmente solteras, con un total del $72,1 \%$. Con respecto a las mujeres casadas, contamos con un $9,3 \%$. No existen datos de mujeres divorciadas y practicantes de RA, y con respecto a otros casos, hay un $18,6 \%$.

\subsection{Nivel de estudios de las practicantes}

No existen datos de mujeres que practiquen y que no tengan estudios. Con estudios primarios, existen un 4,7\%, Secundarios, un 7,0\%, Formación profesional, un $11,6 \%$. Mujeres diplomadas obtienen un total de $14,0 \%$, mientras que universitarias superiores un total de $60,5 \%$. Aquellas mujeres que representan otra situación, solamente hay un $2,3 \%$ de los casos. 


\subsection{Profesión de las practicantes}

Las mujeres son Autónomas en un 26,8\%, micntras que las funcionarias representan el 7,9\%. El mayor valor se da en el cucrpo de docentes de Actividad Física y Educación Física, con un $35,8 \%$, bomberas, con un $4,8 \%$. No existen datos de mujercs practicantes do RA que sean empresarias, terminando con las trabajadoras asalariadas, que cuentan con un $24,7 \%$, obteniendo el $0,0 \%$ para las cstudiantes y otras.

\subsection{Situación actual de las practicantes}

La situación de las mujercs cs bastante resumida puesto que sólo sc agrupan en mujeres que estudian y trabajan a la tex con un total de th $34,9 \%$, y mujeres en situación de activas ocupadas cen un $65,1 \%$.

\subsection{Opinión sobre la participactón masculina y femenim}

A los compctidores,

Tabla 4. Opinión sobre la participación masculina superior a la femenina

\begin{tabular}{|c|c|c|}
\hline & $\mathrm{N}$ & $\%$ \\
\hline $\mathrm{Si}$ & 246 & 88,8 \\
\hline No & 8 & 2,9 \\
\hline Total & 254 & 91,7 \\
\hline
\end{tabular}

les preguntó sobre la opinión quc terian sobre si la participación masculina cra major que la femenina, y la resuesta fue que sí cn un $96,9 \%$ que no $\mathrm{cn}$ un $3,1 \%$. No contesta el $6,5 \%$

\subsection{Motivos de menor participacion femenina}

Con respecto a la pregunta de si el mátivo de menor participación femenina con respecto a la masculina se dcbała kotivos de organización de un raid, se afirmó que sí en un $88,0 \%$ y que jo en un $72,0 \%$. Por motivo de dificultad técnica, sí con $35 \% \%$ yno con $642 \%$; 1 or dificultad táctica, si con $8,2 \%$ y no con $91,8 \%$ por dificulted fisica si con

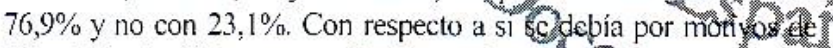
dificultad psicológica, respondieron que si un 1,5\%\$9 youn 98,5\%. Por motivo de cultura deportiva, si un 78,4 y no un $21.6 \%$, pochogtive de educación deportiva de la mujer, rcspondieron que si un $79,3 \%$ y notn $20,7 \%$. Por otros motivos, la respucsta fue que si un $29.0 \%$ y no un $71,0 \%$.

\begin{tabular}{|l|c|c|c|c|}
\hline \multirow{2}{*}{ Tabla 5. Menor participación femenina por diferentes motivos } \\
\hline & \multicolumn{2}{|c|}{$\mathrm{N}$} & \multicolumn{2}{c|}{$\%$} \\
\cline { 2 - 5 } & $\mathrm{Si}$ & $\mathrm{No}$ & $\mathrm{Si}$ & $\mathrm{No}$ \\
\hline Organización de un raid & 21 & 54 & 28,0 & 72,0 \\
\hline Dificultad técnica & 29 & 52 & 35,8 & 64,2 \\
\hline Dificultad táctica & 6 & 67 & 8,2 & 91,8 \\
\hline Dificultad lisica & 83 & 25 & 76,9 & 23,1 \\
\hline Dificultad psicológica & 1 & 67 & 1,5 & 98,5 \\
\hline Cultura deportiva & 87 & 24 & 78,4 & 21,6 \\
\hline Fducación deportiva de la mujer & 88 & 23 & 79,3 & 20,7 \\
\hline Oros motivos & 9 & 22 & 29,0 & 71,0 \\
\hline
\end{tabular}

\subsection{Importancia de la participación femenina}

Los raiders opinan que la participación femenina cn un raid es muy importante, un $53,7 \%$, bastante importante un $22,6 \%$, importante un $21,1 \%$, poco importante un 2,2\% y nada importantc sólo el $0,4 \%$.

\subsection{Aspectos en los que destaca la mujer raider}

Sobre los aspectos en que destaca la mujer raider, se preguntó si eran cn su aspecto fisico y respondieron que si un $42,2 \%$ y no un
$57,8 \%$. En su aspecto táctico, las respuestas representan que si un $59,7 \%$ frente al no un $40,3 \%$. Con respecto a su aspecto técnico, respondicron que si un $47,5 \%$ y no un $52,5 \%$ En relación al aspecto

\begin{tabular}{|c|c|c|}
\hline \multicolumn{3}{|c|}{$\begin{array}{c}\text { Tabla 6. Importancia de la participación } \\
\text { femenina en un equipo de raid }\end{array}$} \\
\hline & $\mathrm{N}$ & $\%$ \\
\hline Muy importante & 145 & 53,7 \\
\hline Bastante importante & 61 & 22,6 \\
\hline Importante & 57 & 21,1 \\
\hline Poco importante & 6 & 2,2 \\
\hline Nada importante & 1 & 0,4 \\
\hline Total & 270 & 97,5 \\
\hline
\end{tabular}
psicológice, respondieronque si un $87,3 \%$ y no un $12,7 \%$, mientras que en reląión a otros aspectoss, afimaron que si un $32,5 \%$ frente al no un $67,5 \%$

\subsection{Requisitos que debe reuhir la mujer raider}

Los raiders respondierom quic los requisitos que deben reunir las mujeres rajders son, iguales que al masculino con un $95,5 \%$, frente a los que eligeren la opción de diferentes al masculino con un $4,5 \%$.

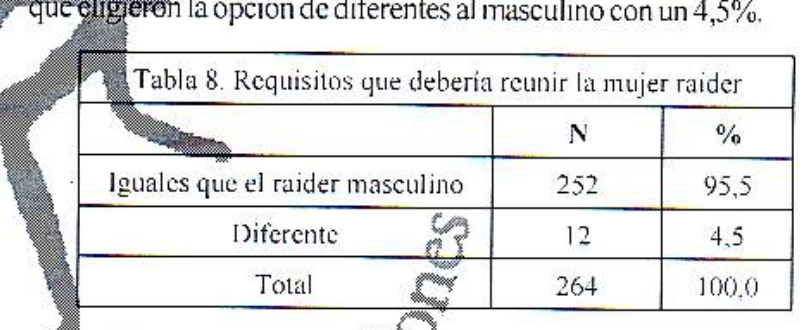

4. Discusión

Relacionados con Jas atudes, Qliyera y Olivera (1998), sitúa a la mayoria de los encucstados en Barcélona en cdades de 15-25 años, seguidos de los cuectuentan entétos 26-35 años de cdad. Al igual que Ged Ferando (1997) en strestudio sobrc las nuevas modalidades deportivas de AFMN donde constata que son los jóvenes los practicantes máshalifurdes, con edades de 16 a 24 años, junto con los comprendífos entre los 25 y 34 años de cdad, seguido de los que tiene cdades entre los 35 y 44 años. Según García Ferrando (1997), sobre los visitantes de Aigües Tortes, los grupos de edades mús importantes son los conmprendidos cntre los 22 y 31 años, seguidos de los 32 a los 41 años, deduciendo que más del $64 \%$ de los visitantes ticne más de 32 años. Granero (2004), cn su análisis sobrc los Senderistas a Santiago, observa como casi la mitad de los encuestados se encucntran con cdades entre los 21 y 40 años de edad, destacando principalnente los comprendidos entre los 21-30 años, mientras que dos de cada die $<$ peregrinos estarían entre los 31 y 40 años. El porcentaje de los comprendidos entre los 41 y 50 años, es del $17.3 \%$, descendiendo a un $14.6 \%$ entre los 16 y 20 años. A lo que se le puede sumar las aportaciones de Miguel et al. (2005), sobre los corredores de Carreras de Aventura, quicnes concluyen que el perfil de edad se sitúa entre los 19 y 43 años, lo que viene a corroborar los resultados obtenidos en nuestro estudio, ya que la franja de edades de mayor practicantes suele rondar los 26 y los 35 años, siendo la gran mayoría menores de 35 años.

A nivel de género Rieu (1986), especifica que dentro del triatlón, cl perfil suele ser un deportista varón. En consonancia con los resultados

\begin{tabular}{|l|c|c|c|c|}
\hline \multirow{2}{*}{ Tabla 7. Aspectos en lo que destaca la mujer raider al competir } \\
\cline { 2 - 5 } & \multicolumn{3}{|c|}{$\mathrm{N}$} & \multicolumn{2}{c|}{$\%$} \\
\hline Asi & No & $\mathrm{Si}$ & No \\
\hline Aspecto fisico & 76 & 104 & 42,2 & 57,8 \\
\hline Aspecto táctico & 123 & 83 & 59,7 & 40,3 \\
\hline Aspecto técnico & 84 & 93 & 47,5 & 52,5 \\
\hline Aspecto psicológico & 186 & 27 & 87,3 & 12,7 \\
\hline Otros aspectos & 13 & 27 & 32,5 & 67,5 \\
\hline
\end{tabular}


mostrados por otros estudios realizados, como los de Miranda, Olivera y Mora (1995), donde los practicantes son en su mayoría hombres, (62.2\% de los casos corresponde a hombres frentes al 37.7\% que responde a las féminas). En esta línea, hay que añadir los trabajos de Olivera y Olivera (1998), Granero (2004), Miguel et al. (2005), junto con Torres (2001), que afirman coincidiendo en su mayoría con un hombre, frente a las mujeres. Y Granero (2004), sobre los senderistas que obtiene que el $61.5 \%$ son hombres frente al $38.5 \%$ que representa a las mujeres. De la Rosa (2007), expone además que para potenciar la participación femenina en los RA, dentro de la Liga Española de RA 2007-2008, en las categorías de Elite y Aventura, los equipos tendrán que puntuar al menos en una de las pruebas corriendo obligatoriamente como equipo mixto. En la categoría de Aventura, las bonificaciones aumentarán del 5\%,10\% al 20\% si corre una raider todo el recorrido o solamente una sección.

En cuanto al estado civil de los raiders y dentro de los trabajos realizados sobre practicantes de AFMN y DA, Granero (2004), expone sus datos coincidiendo con los obtenidos en este trabajo. Así, los raiders y practicantes de estos deportes son en su mayoría personas solteras, existiendo también un gran porcentaje de casados/as (Miguel et al. 2005).

Sobre el nivel cultural de los raiders, se observa en los resultados un nivel cultural bastante alto, ya que la mayoría poseen estudios de Formación Profesional, Diplomados Universitarios y Universitarios Superiores. En esta línea, Olivera y Olivera (1998), afirman que casi la mitad de los practicantes de Barcelona, poseen un nivel cultural elevado. Con Granero (2004), ocurre lo mismo, donde un tercio de los senderistas son universitarios de grado superior, casi otro tercio tienen estudios secundarios de bachillerato (BUP y COU) y el resto con estudios universitarios de grado medio, formación profesional y estudios primarios, destacando que muy pocos deportistas no poseían estudios. Estos datos vienen a confirmar las conclusiones de Miguel et al. (2005), donde afirman que poseen estudios de segundo grado, dando a entender el alto nivel cultural de los deportistas.

Respecto a la situación laboral, a pesar de ser muy variada proliferan los raiders autónomos y docentes de Actividad Física y Educación Física, todos ellos con gran cantidad de tiempo libre para poder entrenar e ir a las competiciones deportivas. Los resultados se encuentran en parte en consonancia con los de Miguel et al. (2005), ya que afirman que los corredores de aventura poseen trabajos de 5 a 8 horas de media, por lo que les deja tiempo libre para entrenar y competir. Por lo que según Torres (2001), la profesión más abundante es la de Funcionario Titulado. Olivera y Olivera (1998), sitúa en su trabajo a la mitad de los encuestados como estudiantes, seguidos de los trabajadores asalariados, amas de casa y los que pertenecen a profesiones liberales y/o están en el paro.

\section{Conclusión}

Los resultados estadísticos a través de la muestra seleccionada establecen que el perfil sociodemográfico de la mujer que practican RA

- Mujeres entre los 26 y 35 , siendo preferentemente de los 31 a los 35 años de edad. Lo cual nos indica que estos deportes son practicados por mujeres relativamente jóvenes.

- La población femenina es considerada como poco practicante, sobre todo si la comparamos con los hombres, que suelen ser los que más participan en este tipo de pruebas.

- El estado civil de estas competidoras suele coincidir con mujeres solteras, indicándonos también que pueden poseer de bastante tiempo libre para entrenar y competir.

- En cuanto a nivel de estudios, son mujeres con niveles de Universidad Superior y Diplomadas Universitarias, lo que nos indica el alto grado de instrucción de estas deportistas.

- La profesión tiende a ser de docente en Actividad Física y Educación Física, seguidas de autónomas o asalariadas, con lo que la mayoría son activas ocupadas y en algunos casos, estudian y trabajan.
- Los raiders opinan que la participación femenina es mucho menor que la masculina, debido a la educación deportiva de la mujer, seguido de la cultura deportiva y por último, debido a la dificultad física de los raids.

- La participación femenina en estas pruebas se considera como muy importante.

- La mujer destaca fundamentalmente en su aspecto psicológico y táctico dentro de las competiciones.

- Por último comentar que se concluye con la opinión de que los requisitos que debería reunir la mujer raiders son los mismos que poseen los raiders masculinos.

\section{Referencias}

Aguilera, M. (2004). Mónica Aguilera y Nuria Domínguez ganadoras del II Raid WIAR Salomón 2004. http://www.desnivel.com [Consulta: 6 Julio 2004].

Arias, R. (2005). Año de estreno. Campeonato de España de Raids. Revista Outdoor, (4) 16-21.

Baigorria, A. y Fernández, R. (1996). Sociología del deporte en España. En M. García y M. Martínez (eds), Ocio y Deporte en España: Ensayos sociológicos sobre el cambio (pp.25-36). Valencia: Tirant lo Blanch.

Bustillo, CH. (2005). Preparado para triunfar: Raids, el asalto a la competición. Revista Outdoor, (3).

Canales, I., Lagardera, F. y Perich, M.J. (1995). Lo femenino en las actividades físicas en la naturaleza. Apunts. Educación Física y Deportes (41) 141-150.

Canales, I. y Perich, M.J. (2000). Sensaciones y vivencias de la mujer en la práctica de actividades física en la naturaleza. Revista de Educación Física. Renovar la teoría y la práctica, (79) 37-43.

De La Rosa, A. (2007). Liga Española de Raids de Aventura. Todo está listo. Revista FinisherTriatlon (74), 56-57.

García Ferrando, M. (1997). Los españoles y el deporte, 1980-1995. Un estudio sociológico sobre comportamiento, actitudes y valores. Madrid: Consejo Superior de Deporte y Tirant lo Blanch.

Gutiérrez, M. y Oña, A. (2005). Metodología en las ciencias del deporte. Madrid: Síntesis.

Granero, A. (2004). Expectativas y vivencias en la actividad fisicodeportiva del peregrino. Un antes y un después en el Camino de Santiago. Tesis Doctoral, Universidad de Almería, Almería.

Herrera, J.R. y Pilatti, L.A. (2005). O nicho 'Esportes de Aventura': um procsso de civilizaçáo ou descivilizaçáo?». Revista Digital- [en línea], $N^{\circ}$ 87. Buenos Aires. Disponible en: http.//www.efdeportes.com. [Consulta realizada el 12 de marzo de 2006].

Martos, C. (1999). Contributos para a compreensao dos desportos radicls. Revista de Ciencias do Desporto. Ludens. (Volumen 16, 2) 53-63.

Martos, M. (2004). Entrevista al Equipo Grandes Espacios. http:// www.aventur.es [Consulta: 15 Julio 2006].

Miguel, D., Andrade, A. y Portela, A. (2005). Caracterizaçäo do perfil sócioeconômico, motivacional, stress e ansiedade percibidos de competidores de corridas de aventura. Revista Digital- [en línea], $N^{\circ} 90$. Buenos Aires. Disponible en: http.//www.efdeportes.com. [Consulta realizada el 2 de febrero de 2005].

Miranda, J., Olivera, J. y Mora, A. (1995b). Análisis del ámbito empresarial y de la difusión sociocultural de las actividades físicas de aventura en la naturaleza. Apunts. Educación Física y deportes (45), 130-137

Olivera, A. y Olivera, J. (1998). Análisis de la demanda potencial de las actividades físicas de aventura en la naturaleza en la ciudad de Barcelona. Apunts. Educación Física y Deportes (52) 92-102.

Pinos, M. (1997). Actividades y juegos de Educación Física en la Naturaleza. Guía práctica. Madrid: Gymnos.

Rebollo, S. y Feriche, B. (2002). Problemática medioambiental y práctica deportiva. Revista Digital- [en linea], $N^{\circ} 45$. Buenos Aires. Disponible en: http.//www.efdeportes.com. [Consulta realizada el 15 de marzo de 2003].

Rieu, M. (1986). El coste energético del triatlón. Una reflexión preliminar. Revista Española de Educación Física y Deporte, (12), 40-42.

Roca, E. (2005). Mujeres y raids de aventura. Revista Finisher Triatlón, (58).

Sierra, R. (2001). Técnicas de investigación social. Teoría y ejercicios. Madrid: Paraninfo Thomson Learning.

Torres, E. (2001). Perfil del turista deportivo en Granada. En M. Latiesa, P. Martos y J.L. Actas del congreso Deporte y cambio social en el umbral del siglo XXI (pp. 275-289). Madrid: Ed. AEISAD. 